\title{
Algunos comentarios para evaluar el primer año del Plan Auge en el sistema público de salud
}

RAFAEL URRIOLA(1)

\section{INTRODUCCIÓN}

Este documento tiene por objeto pasar revista -de un modo esencialmente descriptivo- a los nodos críticos que surgen con la Ley 19.966 que da curso legal al Plan de Acceso Universal con Garantías Explícitas (Auge) en el sistema público, especialmente en cuanto a las garantías explícitas en: acceso, oportunidad (plazos máximos), calidad (según protocolos públicos)* $\mathrm{y}$ financieras (costos y topes máximos conocidos). En consecuencia, el objetivo central es entregar información acerca de lo sucedido en este primer año de implantación del Auge.

Es evidente que un desafío de este tipo para un sistema de salud como el chileno que absorbe alrededor del $6 \%$ de los recursos nacionales (en el que, el sistema público se hace cargo de dos tercios de la población), ha implicado serias transformaciones institucionales y culturales, de las cuales, sin duda, el carácter de exigibilidad para un determinado número de prestaciones resulta un ejemplo inédito en países de menor desarrollo relativo como Chile. Por ello, el Auge conviene verlo como un proceso que está en constante perfeccionamiento y que, en todo caso, como se verá, es valorado favorablemente por la ciudadanía.

\section{GARANTIA DE ACCESO}

La Garantía Explícita de Acceso es la obligación del Fondo Nacional de Salud (Fonasa) y de las Instituciones de Salud Previsional (Isapre), de asegurar el otorgamiento de las prestaciones de salud garantizadas a los beneficiarios de las leyes $\mathrm{N}^{\circ} 18.469$ y $\mathrm{N}^{\circ} 18.933$, respectivamente. Estas fueron explicitadas en enero de 2005 mediante el Decreto Supremo $\mathrm{N}^{\mathrm{o}} 170$, que aprueba 25 problemas de salud. A partir del $1^{\circ}$ de julio de 2006 entraron en vigencia otros 15 problemas para completar los 40 actualmente garantizados.

Tienen derecho a tales garantías todos los afiliados a los sistemas de Isapres y al Fonasa.

Este último, a diciembre de 2005, contaba con 11.120.094 beneficiarios. La Figura 1 muestra la participación de la población, según seguro de afiliación en la que se destaca que un $12,6 \%$ de la población (alrededor de dos millones de personas) quedaría marginado de los beneficios del Plan Auge por no pertenecer a los sistemas en que éste es obligatorio.

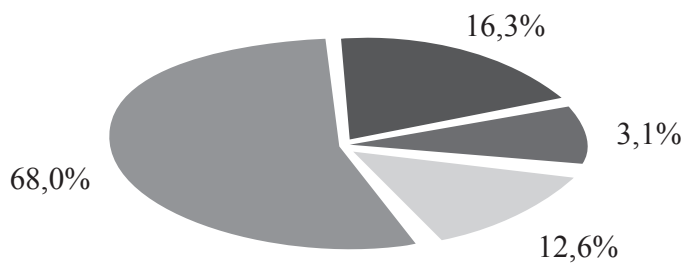

FONASA ISAPRE IFFA OTROS

Figura 1. Proporción de la población según afiliación a seguros de salud.

Fuente: Subdepartamento de Estadísticas y Red de Conocimiento. Fonasa.

\footnotetext{
(1) Jefe Departamento de Planeamiento Institucional. Fonasa. rurriola@fonasa.gov.cl

* Los reglamentos de acreditación y certificación que requerirán prestadores personales e institucionales, respectivamente, aún no han sido aprobados legalmente, razón por la cual esta garantía no se examina en este artículo.
} 
La garantía de acceso, principalmente se refiere a una opción institucional, es decir, a que el sistema público de salud no rechace -por cualquier circunstancia- a quienes tienen derecho dada su correcta afiliación a este seguro. En los hechos, sólo deben quedar sin acceso las personas que pertenecen a otros sistemas o quienes no estén afiliados a sistema alguno. En la medida que esta proporción de la población alcanza a cerca del $12 \%$. Fonasa decidió desarrollar campañas que conciten mayor regularización en los pagos de cotizaciones a los trabajadores dependientes y desplieguen información de las garantías a las que pueden acceder los trabajadores independientes.

En todo caso, en los 25 problemas Auge vigentes hasta el $1^{\circ}$ de julio del 2006, se han atendido cerca de dos millones de personas, lo que es compatible con las expectativas preliminares estimadas $\mathrm{y}$, de paso, muestra que el sistema público de salud en general no ha colapsado, pese a la mayor demanda que se preveía para resolver estos eventos.

Cabe notar que, en el transcurso del año de vigencia del Auge se han producido algunas diferencias entre los casos atendidos y las estimaciones preliminares, las cuales, en el caso de la atención primaria -con menos casos que los estimados- se atribuye a dificultades en la actualización de los sistemas de registros. En cambio, en cataratas y prematurez, que corresponden a la atención en el nivel secundario, se detectan más casos que los estimados, lo cual requerirá revisiones, sea en las prevalencias determinadas con anterioridad o en los mecanismos de registros actuales. Aún así, lo relevante es que, en el período, según estadísticas de Fonasa, sólo se han registrado 367 reclamos relacionados con la garantía de acceso, de los cuales, el $97 \%$ fueron resueltos satisfactoriamente y ninguno ha requerido usar otras instancias, porque los 14 restantes están en calidad de pendientes.

\section{GARANTIA DE OPORTUNIDAD}

La Garantía Explícita de Oportunidad que otorga el Plan Auge es el plazo máximo para el otorgamiento de las prestaciones de salud garantizadas, en la forma y condiciones que determina la ley. Dicho plazo considerará, a lo menos, el tiempo en que la prestación deberá ser otorgada por el prestador de salud que corresponda en primer lugar; El tiempo para ser atendido por un prestador distinto, designado por Fonasa o la Isapre, cuando no hubiere sido atendido por el primero; $y$, en defecto de los anteriores, el tiempo en que el prestador definido por la Superintendencia de Salud deba otorgar la prestación con cargo a las instituciones antes señaladas. No se entenderá que hay incumplimiento de la garantía en los casos de fuerza mayor, caso fortuito o que se deriven de causa imputable al beneficiario.

Considerando las mayores exigencias que resultan del Plan Auge ha sido necesario depurar los procesos que conducen a una creciente eficiencia en lo concerniente a Monitoreo, Control y Fiscalización que permitan hacer un adecuado seguimiento de los problemas contenidos en el Régimen de Garantías de Salud para mejorar la eficiencia general del sistema.

Estas actividades están permitiendo aproximarse paulatinamente a lograr tres objetivos:

a) Verificar la confiabilidad de los datos incorporados al Sistema de Información que registra las múltiples actividades definidas para cada uno de los problemas de salud Auge.

b) Asegurar el cumplimiento de la Garantía de Oportunidad en los términos establecidos para cada uno de los problemas Auge.

c) Desarrollar una herramienta para evaluar el comportamiento de la oferta, en cuanto a gestión de las redes asistenciales.

Basado en el sistema de Información de Salud, se han definido hitos calificados en: Alerta Temprana, que es el primer nivel del proceso de control y evidencia el estado de la garantía de oportunidad en el equivalente de cumplimiento del 50\% de los tiempos establecidos; y Alerta Final o Roja, es decir, el segundo nivel de control que se "gatilla" cuando el estado de la garantía de oportunidad llega al $80 \%$ del tiempo establecido. Las Alertas Rojas se han definido con el objetivo de tomar medidas correctivas que anticipen al Riesgo de Incumplimiento. En el 
período alcanzaron a 7.119 , concentradas en cataratas e hipertensión lo cual, sin embargo, representa apenas $0,36 \%$ del total de casos registrados en los doce meses transcurridos.

Llegando a esta fase del proceso, si es pertinente, la autoridad desencadena procesos de gestión alternativa de compra. Este proceso tensiona al máximo al conjunto del sistema porque exige definiciones precisas de cada uno de los problemas del Plan Auge, a objeto de verificar las condiciones de entrega (capacidad de oferta pública y privada en segunda instancia) de las prestaciones.

Todo lo anterior ha conllevado un enorme esfuerzo en cuanto a tecnologías de información; disponibilidad de recursos humanos y tecnológicos; capacitación del personal en estas nuevas funciones; y, diseño de Programa de Supervisión y Apoyo a la Gestión de Controles Regionales.

Uno de los factores decisivos para el cumplimiento de las garantías de salud, es la capacidad de anticipar situaciones críticas de tal modo que las "brechas" sean cubiertas oportunamente mediante convenios con el sector privado cuando la oferta pública no sea suficiente en concordancia con las facultades que le confiere la Ley $\mathrm{N}^{\mathrm{o}} 19.650$.

En consecuencia, la función de comercialización y compra que se desarrolla al momento de detectar carencias, requiere asimismo, de una afinada coordinación entre los Servicios de Salud, la Subsecretaría de Redes y Fonasa para lo cual se han creado los Directorios de Compra.

Tabla 1. Convenios de compra de prestaciones (julio 2005- julio 2006)

\begin{tabular}{|c|c|c|c|}
\hline \multicolumn{4}{|c|}{ CONVENIOS VIGENTES } \\
\hline $\begin{array}{l}\text { Endoprótesis de } \\
\text { cadera }\end{array}$ & Hospital DIPRECA & Trato Directo & Nacional \\
\hline Escoliosis & Hospital FACH & Trato Directo & Nacional \\
\hline $\begin{array}{l}\text { Implantes } \\
\text { Cocleares }\end{array}$ & Hospital Naval & DFL 36 Centralizado & $\begin{array}{l}\text { Nacional, red } \\
\text { para I, II, III, IV, } \\
\text { VIII y XII Región. }\end{array}$ \\
\hline $\begin{array}{l}\text { Tratamientos } \\
\text { Hemodiálisis }\end{array}$ & $\begin{array}{l}142 \text { Centros en Convenios } \\
\text { el año } 2005 \text { a nivel país; } \\
176 \text { Centros en Convenio } \\
\text { Marco el } 2006\end{array}$ & $\begin{array}{l}\text { Licitación Pública } \\
\text { Directa FONASA el } \\
2005 \text { y Convenio } \\
\text { Marco el } 2006\end{array}$ & Nacional \\
\hline $\begin{array}{l}\text { Tratamientos } \\
\text { Peritoneodiálisis }\end{array}$ & $\begin{array}{l}43 \text { Centros en Convenios } \\
\text { el año } 2005 \text { a nivel país; } \\
176 \text { Centros en Convenio } \\
\text { Marco el } 2006\end{array}$ & $\begin{array}{l}\text { Licitación Pública } \\
\text { Directa FONASA el } \\
2005 \text { y Convenio } \\
\text { Marco el } 2006 \\
\end{array}$ & Nacional \\
\hline Radioterapia & FALP & Trato Directo & Nacional \\
\hline Cataratas & Kychenthal y Asoc. & Trato Directo & I, II y III Región \\
\hline Cataratas & $\begin{array}{l}47 \text { prestadores a nivel } \\
\text { nacional }\end{array}$ & $\begin{array}{l}\text { Convenios Marco } \\
\text { Chilecompras }\end{array}$ & Nacional \\
\hline $\begin{array}{l}\text { Accesos Vasculares } \\
\text { para hemodiálisis }\end{array}$ & 20 prestadores a nivel nacional & $\begin{array}{l}\text { Convenios Marco } \\
\text { Chilecompras }\end{array}$ & Nacional \\
\hline Radioterapia & $\begin{array}{l}\text { Centro de Especialidades } \\
\text { Médicas CEM-IRAM Santiago } \\
\text { e Instituto Oncológico de } \\
\text { Viña de Mar año } 2005 \text { y } 10 \\
\text { prestadores a nivel nacional }\end{array}$ & $\begin{array}{l}\text { Trato Directo año } \\
2005 \text { y Convenios } \\
\text { Marco Chilecompras }\end{array}$ & Nacional \\
\hline $\begin{array}{l}\text { Cardiopatías } \\
\text { Congénitas }\end{array}$ & $\begin{array}{l}\text { Hospital Clínico Universidad } \\
\text { Católica }\end{array}$ & $\begin{array}{l}\text { Protocolo de acuerdo } \\
\text { de precios }\end{array}$ & $\begin{array}{l}\text { Nacional, red } \\
\text { para Maule, } \\
\text { Llanchipal y } \\
\text { Suroriente RM }\end{array}$ \\
\hline
\end{tabular}


La política de compra de Fonasa (según documento interno del Departamento de Comercialización), es privilegiar- en primer lugarla oferta pública disponible por parte del Sistema Nacional de Servicios de Salud y los establecimientos municipalizados de atención primaria y, en coordinación con el Minsal, propender a la optimización de la oferta de éstos.

Le corresponde a Fonasa realizar anualmente un proceso de evaluación con los establecimientos públicos de salud, representados por los Directores de los Servicios de Salud y Establecimientos Experimentales en su calidad de gestores de red, con el objeto de determinar las "brechas" de oferta $\mathrm{y}$, consecuentemente, definir los recursos que requerirá el sector público y las compras a prestadores privados. Estas, en general, no superan el $10 \%$ de los requerimientos en los problemas incluidos en este primer año de funcionamiento del Plan.

Los contratos con prestadores públicos contemplan tanto la resolución en sus propios establecimientos, como la compra a terceros que éstos directamente realizan. Con fecha 31 de enero del 2005 y 31 de enero del 2006, Fonasa suscribió convenios con los 28 Servicios de Salud y 3 establecimientos experimentales para todas las prestaciones GES.

Complementariamente -a modo de ejemplose realizaron, entre otros, los siguientes procesos de compra a prestadores "extra-sistema": No obstante, se debieron realizar otras compras a prestadores privados ante riesgo de incumplimiento, realizadas por los Servicios de Salud y analizadas en el marco de los Directorios de Compra. Si bien, las situaciones ante riesgo de incumplimiento de la garantía de oportunidad, se resolvieron mayoritariamente dentro del sector público, empezaron a ser más importantes las compras a privados en el año 2006, especialmente la intervención quirúrgica de cataratas (981 cirugías por un total cercano a los 432 millones de pesos).
No cabe duda de que las garantías de oportunidad representan la mayor complejidad y desafío de la Reforma de Salud, tanto por la amplitud de posibilidades de ocurrencia de los fenómenos sanitarios, como por la imprescindible necesidad de constituir redes afiatadas en el sistema de salud público de tal modo que las derivaciones de pacientes sean efectivas y oportunas. Asimismo, pequeños errores en los sistemas de registros pueden provocar resultados estadísticos erróneos con sus secuelas sobre el cumplimiento de las garantías.

Teniendo en consideración tales problemas los resultados de reclamos sobre garantías de oportunidad en Fonasa se ubican en un rango razonable. De un total de 475 reclamos calificados como no cumplimiento de la garantía de oportunidad, sólo 7 quedaron pendientes en el año de vigencia del Plan Auge. Por cierto, el relativo desconocimiento de los derechos de las personas puede influir en esta baja cifra, pero a la vez que las personas podrán aumentar sus demandas gracias a la mayor información que el propio sistema público está otorgando, los Servicios de Salud irán aumentando la eficiencia $\mathrm{y}$, en consecuencia, reduciendo las causas de los reclamos.

\section{GARANTIA FINANCIERA}

La Garantía Explícita de Protección Financiera es la contribución que deberá efectuar el afiliado por prestación o grupo de prestaciones, la que deberá ser de un máximo de $20 \%$ del valor determinado en un arancel de referencia del Régimen.

Si bien, en los inicios del debate acerca del financiamiento del Auge se hizo alusión a los aportes que harían los propios afiliados por concepto de copagos en las prestaciones otorgadas por el Auge, éstos se situaban en los

\footnotetext{
** El marco presupuestario para el Auge, definido inicialmente por Hacienda en memorándum del 5 de marzo de 2002 de Mario Marcel a Dr. Hernán Sandoval, alcanzaba a 515,5 mil millones de pesos, incluyendo lo asignado en el presupuesto del 2002 al Programa de Prestaciones Valoradas, Atención Primaria, copagos y una expansión de 133,8 mil millones. Este marco se utilizó para construir la propuesta a los Ministros y el Presidente el 4 de abril de 2002. *** R. Bitrán. Verificación del costo esperado por beneficiario del conjunto priorizado de problemas de salud con garantías explícitas. Minsal, noviembre 2005.
} 
alrededores del 5\% de los costos estimados totales, especialmente porque un alto porcentaje de los problemas atendidos en Auge se espera corresponda a los grupos A y B que están exentos de copago, así como los adultos mayores. Aún así, esta suma sólo podrá recaudarse en tanto se mejoren los mecanismos de cobro a quienes actualmente se atienden en los servicios públicos o teniendo derechos a ello, y en cuanto se establezcan mecanismos más estrictos de copago.

\section{EL FINANCIAMIENTO DEL PLAN AUGE}

Las estimaciones iniciales de los costos de los 56 problemas Auge se situaron en 3,06 Unidades de Fomento (UF) promedio por persona. Este cálculo fue concordado entre el Ministerio de Hacienda y el de Salud. El costo total estimado para el sector público, en base a la población del año 2002, alcanzó a 513.613 millones de pesos. En términos per cápita esto representó cerca de $\$ 50.000$ por beneficiario al año**. Recordemos que esta cifra fue reevaluada a fines del $2005^{* * *}$ y los resultados obtenidos se aproximaron al valor inicial.

Al precio de la UF en julio de 2006 y con 40 problemas (2,04 UF), el costo de ellos se sitúa en alrededor de 400.000 millones de pesos. Los valores del presupuesto de salud, administrado por Fonasa en 2005, fueron de $\$ 1.496 .396$ millones y, en 2006, se asignaron $\$ 1.713 .342$ millones, es decir, un incremento de $14,2 \%$. Esto permite concluir que el Auge sólo ocupa el 23\% del presupuesto general sectorial destinado a prestaciones. Por otra parte, como se observa en el cuadro siguiente, los aportes globales al presupuesto de salud en los últimos cinco años han aumentado en 58\%. Lo que es más importante es que los aportes adicionales absolutos (627.000 millones de pesos), han sido ampliamente suficientes para financiar la totalidad del Auge y, además, restan excedentes para resolver otros acuciantes problemas de salud.

\section{ACERCA DE LA SATISFACCION DE LOS BENEFICIARIOS}

En enero de 2006, la Superintendencia de Salud
Tabla 2. Balances Fonasa (2001-2006) (millones de pesos)

\begin{tabular}{|ll|}
\hline 2006 & 1.713 .342 \\
\hline 2005 & 1.496 .396 \\
\hline 2004 & 1.330 .670 \\
\hline 2003 & 1.201 .177 \\
\hline 2002 & 1.115 .062 \\
\hline 2001 & 1.087 .254 \\
\hline
\end{tabular}

Fuente: Boletines Estadísticos Fonasa (varios años) e informes financieros.

evaluó los casos Auge a fin de sondear la percepción obtenida respecto de los beneficios recibidos, trámites efectuados y el grado de satisfacción alcanzado. El Superintendente afirmó que “... si bien los resultados de la fiscalización son bastante positivos respecto a la satisfacción que muestran, señaló como preocupantes el alto nivel de desinformación que tienen los usuarios del Fonasa..." No obstante, el propio Superintendente, Dr. Manuel Inostroza, señaló "que el desconocimiento en salud pública se debe a que los integrados a Fonasa son ingresados automáticamente a esta red". El informe también destacó que "Consultados por los pagos de las prestaciones, el 93\% de los usuarios de Fonasa se beneficia con la gratuidad de éstas". Asimismo, la encuesta barómetro del CERC de agosto de 2006, indica que en el año "una amplia mayoría de los chilenos, 59\%, califica con buena nota a su sistema de previsión de salud y disminuye 11 puntos quienes la califican con mala nota (20\%). Luego indica que "se mantiene la buena imagen del Plan Auge... La mitad de los chilenos -51\%cree que con el Auge se "reducen las desigualdades de acceso a la salud".

\section{CONCLUSIONES}

No cabe duda de que el proceso de Reforma de la Salud chileno es de una enorme complejidad y que requiere transformaciones profundas en la cultura institucional de trabajadores y usuarios del sector. Este proceso está en marcha y deberá perfeccionarse tanto en calidad como en amplitud en los meses que 
vienen, porque ahora se han incorporado 15 nuevos problemas para completar 40 en 2006; 56 en 2007 y 80 hasta fines de 2010 según se aseguró en el programa presidencial vigente.

Más allá de las desconfianzas generadas por el Plan -especialmente en el proceso de debate previo a su implementación- el Auge ha cubierto problemas de cientos de miles de personas y eso no está en duda. Asimismo, se han destinado ingentes recursos para satisfacer la demanda con oferta propia del sector público lo cual se ha logrado en la mayoría de los casos en proporciones altas. Tal esfuerzo ha requerido optimizar la red pública general, lo cual es un proceso que también deberá ir mejorando en el futuro para reducir las alertas y situaciones que puedan comprometer el cumplimiento de las garantías.
En fin, pese a todo, no hay evidencias de que el Auge haya desplazado la oferta de otras patologías en el sector público, en primer lugar, porque sólo ocupa el 23\% del Presupuesto sectorial que, por lo demás, se ha incrementado de manera significativa en los últimos años. En segundo lugar, porque algunos de los problemas Auge se estaban cubriendo con anterioridad como, por ejemplo, aquellos relacionados con infecciones respiratorias agudas o los tratamientos de hemodiálisis para diabéticos.

De cualquier modo, el Plan conlleva una reingeniería importante en la gestión de procesos y en la capacitación en nuevas competencias de los funcionarios, lo cual deberá perfeccionarse gradualmente -pero con rapidez- para evitar problemas en asegurar las garantías, especialmente de oportunidad. 